\title{
Maduración in vitro de ovocitos humanos: una opción para preservar la fertilidad de las mujeres. Reporte del primer caso realizado en Chile
}

\author{
Carolina Ortega-Hrepich ${ }^{1}$, Luis Guzmán M. ${ }^{2}$, Begoña Argüello ${ }^{1}$, Ricardo Pommer T. ${ }^{1}$ \\ ${ }^{1}$ Unidad de Medicina Reproductiva, Clínica Monteblanco, Santiago, Chile. ${ }^{2}$ Grupo de Reproducción Asistida PRANOR, \\ Lima, Perú.
}

\section{RESUMEN}

La maduración in vitro (MIV) de ovocitos humanos es parte de las técnicas de fecundación in vitro, que consiste en la aspiración de ovocitos inmaduros. Es ofrecida principalmente a pacientes con alto riesgo de desarrollar un síndrome de hiperestimulación ovárica, especialmente pacientes con síndrome de ovario poliquístico. Adicionalmente, es un método ofrecido a las pacientes oncológicas para preservar su fertilidad. Presentamos el primer caso de MIV realizado en Chile, en la Unidad de Medicina Reproductiva de Clínica Monteblanco, en una paciente con sospecha de resistencia ovárica a la FSH y quien, con el consentimiento de sus padres, realizó este procedimiento para preservar su fertilidad a futuro.

\section{PALABRAS CLAVE: Maduración in vitro de ovocitos, preservación de fertilidad, síndrome de ovario poliquístico, síndrome de hiperestimulación ovárica}

\section{SUMMARY}

Oocyte in vitro maturation (IVM) is an assisted reproductive technology in which immature oocytes are retrieved from antral follicles. It has been applied mainly in patients with an increased risk of ovarian hyperstimulation syndrome, particularly in patients with polycystic ovarian syndrome. Moreover, it has been proposed as an alternative approach for fertility preservation for oncological patients. We report the first IVM case in Chile, preformed at the Centre for Reproductive Medicine at Clínica Monteblanco, in a patient with suspected ovarian resistance to $\mathrm{FSH}$, who with the consent of her parents, performed IVM to preserve her fertility.

\section{KEY WORDS: Oocyte in vitro maturation, fertility preservation, polycystic ovarian syndrome,} ovarian hyperstimulation syndrome

\section{INTRODUCCIÓN}

La maduración in vitro de ovocitos humanos (MIV) consiste en obtener ovocitos maduros a partir del cultivo in vitro de ovocitos inmaduros; los que son recuperados desde folículos antrales por vía transvaginal luego de una estimulación ovárica nula o mínima (1). Los ovocitos inmaduros recuperados son cultivados con el fin de reiniciar la meiosis y fecundarlos in vitro.

La MIV se ofrece principalmente a pacientes con alto riesgo de desarrollar un síndrome de hiperestimulación ovárica $(\mathrm{SHO})$ como las pacientes con síndrome de ovario poliquístico (SOP). Adicionalmente, 
es un método que puede ser ofrecido a las pacientes oncológicas quienes desean preservar su fertilidad y que deben iniciar radio/quimioterapia de forma inmediata.

Debido a que la aspiración folicular puede ser realizada tanto en la fase folicular como en la fase lútea se puede realizar un ciclo de MIV en diferentes etapas del ciclo menstrual. A continuación, reportamos el primer caso exitoso de MIV realizado en Chile en una paciente con una posible resistencia ovárica a la FSH y quien, con el consentimiento de sus padres, se realizó este procedimiento para preservar su fertilidad futura.

\section{Caso clínico}

Paciente de 15 años con antecedente de pubertad precoz, tratada con acetato de leuprolide desde los 8 años por 2 años. Cinco años después de la menarquia, presentó ciclos de amenorrea, tratados con progesterona para inducir menstruación.

El análisis inicial reveló: FSH 118,5 $\mathrm{mUl} / \mathrm{mL}$; estradiol $25 \mathrm{pmol} / \mathrm{mL}$; AMH 6,82; anticuerpos antinucleares 1:640, 1:80 patrón moteado; anticuerpos antitiroglobulina 23,3; antiTPO 29,3; cariograma 46 $\mathrm{XX}$; síndrome de $\mathrm{X}$ frágil negativo. Ecografía transrectal reveló 25 folículos antrales.

Debido a FSH elevada y recuento de folículos antrales normal para la edad, se sospecha una resistencia ovárica a la FSH. La paciente desea preservar su fertilidad pero debido a la resistencia ovárica a la $\mathrm{FSH}$, un tratamiento convencional de FIV no será adecuado para la estimulación ovárica y aspiración de ovocitos maduros. Se discute el caso con la paciente y con sus padres y se sugiere criopreservar ovocitos utilizando la técnica de MIV.

La paciente se realiza un primer ciclo de MIV, estimulando con $150 \mathrm{UI}$ de r-FSH por 3 días, e induciendo maduración final con 5000 UI hCG. La aspiración folicular se realizó 36 horas post-hCG, sin complicaciones. Se obtienen 14 ovocitos en estadío de vesícula germinal (VG) (Figura 1) que fueron madurados en medio de cultivo.

A las 30 horas de cultivo in vitro, se identifica un ovocito maduro que es criopreservado (Figura 2). Los demás ovocitos se reevalúan a las 48 horas, identificando otros 3 ovocitos maduros, que son criopreservados.

Un mes después la paciente se realiza un segundo ciclo de MIV, administrando únicamente 5000 Ul de hCG 36 horas antes de la aspiración folicular. De los 14 ovocitos recuperados en VG, 4 maduraron in vitro y fueron criopreservados. De este modo, la paciente ha criopreservado 8 ovocitos, los cuales serán desvitrificados y micro inyectados cuando la paciente desee embarazarse.

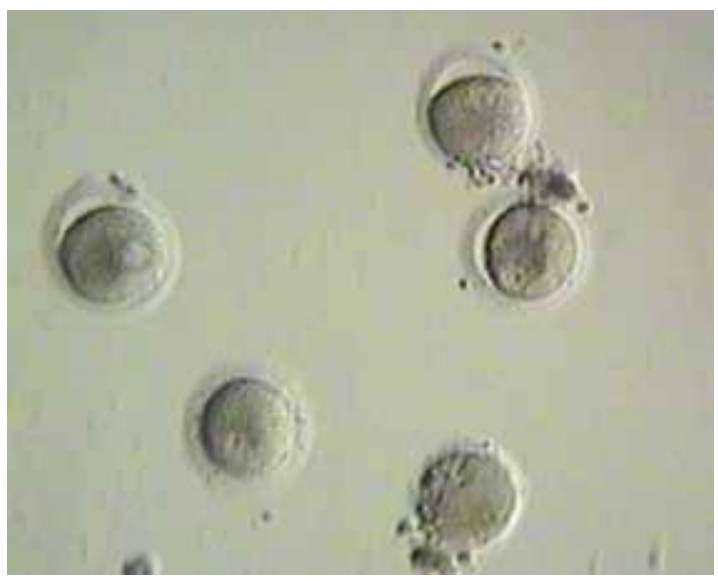

Figura 1. Ovocitos inmaduros (vesícula germinal).

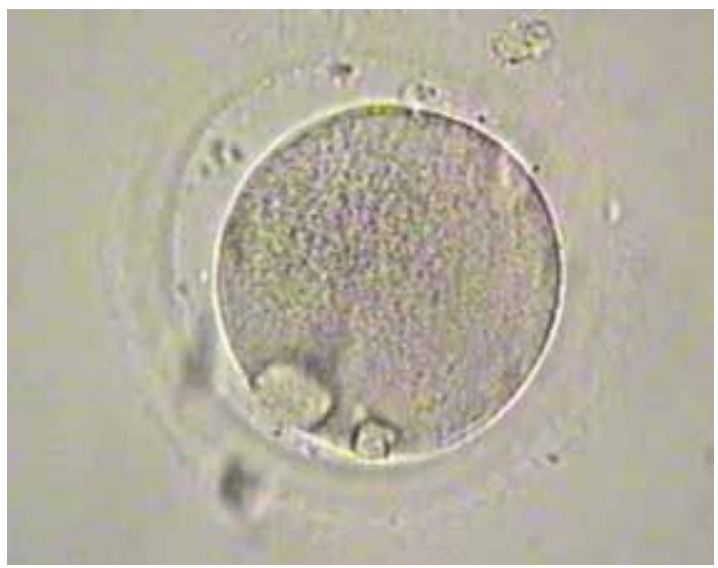

Figura 2. Ovocito maduro (metafase II).

\section{DISCUSIÓN}

Actualmente, la estimulación ovárica controlada con gonadatrofinas exógenas es el pilar fundamental para la obtención de ovocitos maduros durante los tratamientos de FIV. Sin embargo, el uso de gonadotrofinas con el consecuente desarrollo de múltiples folículos, puede provocar SHO (2).

Entre las estrategias para evitar el SHO, la MIV provee la mejor alternativa ya que disminuye el riesgo a un $0 \%$ (3-5). Por este motivo, la MIV está principalmente dirigida a pacientes con alto riesgo de desarrollar SHO, como el SOP (6-12). Las pacientes con ciclos menstruales regulares también se benefician $(13,14)$, como pacientes oncológicas 
que desean preservar su fertilidad (15). Basados en la teoría de Baerwald y cols, quienes sugieren que existiría más de una ola de crecimiento folicular durante el ciclo menstrual y que el crecimiento de folículos antrales podría iniciarse y continuarse en cualquier momento del ciclo menstrual (16), Demirtas y cols y Maman y cols demostraron que ovocitos inmaduros aspirados durante la fase lútea eran capaces de madurar in vitro $(17,18)$. Por lo tanto, pacientes oncológicas quienes desean preservar su fertilidad, pueden aspirarse ovocitos inmaduros en cualquier fase del ciclo ovárico para luego realizar una MIV, sin retardar el inicio de su tratamiento.

El protocolo de estimulación dependerá del diagnóstico de la paciente, del centro y, principalmente de la experiencia del equipo tratante. La diferencia más importante radica en el uso de hCG. Al no administrar hCG se evita la reactivación prematura de la meoisis manteniéndose la comunicación entre las células del cúmulo y el ovocito (19) obteniendo así, una cohorte de ovocitos aspirados totalmente homogénea. Por lo tanto, todos los ovocitos madurarán in vitro al mismo tiempo y en el caso de realizar una inyección intracitoplasmática de espermio, el procedimiento será realizado en un único momento. Sin embargo, al no administrar hCG, la receptividad endometrial está aparentemente comprometida.

De forma contraria, al administrar hCG se pierde la comunicación entre el cúmulo y el ovocito obteniendo diferentes poblaciones de ovocitos, aquellos que maduraron in vivo y aquellos que deberán madurar in vitro en los medios de cultivo de MIV, lo que conllevará a que la inseminación se realice en tiempos diferentes.

Igualmente, la aspiración folicular puede realizarse sin ningún tipo estimulación. Este protocolo es especialmente útil en pacientes oncológicas ya que no hay exposición a gonadotrofinas.

Los resultados clínicos de MIV dependen principalmente del uso o no de hCG antes de la aspiración folicular. En pacientes con SOP a las cuales se les administró hCG previa aspiración folicular, $y$ en quienes se transfirieron embriones derivados de VG y de ovocitos madurados in vivo, Son y cols reportan una tasa de embarazo clínico de $23,3 \%$ y $40 \%$ respectivamente $(20)$.

En pacientes con SOP en quienes no se administró hCG, De Vos y cols reportan una tasa de embarazo con transferencia embrionaria en ciclos frescos inferiores al 10\%, la cual aumenta de forma significativa al transferir embriones desvitrificados en ciclos diferidos con preparación endometrial artificial de $9,4 \%$ a $31,8 \%(p=0,033)(6)$, y de $5,4 \%$ a $37,5 \%(p=0,004)(21)$.

Este resultado fue confirmado por OrtegaHrepich y cols al publicar los resultados clínicos en pacientes con SOP en quienes se transfirieron embriones derivados de ciclos de MIV sin hCG, los cuales fueron todos vitrificados y transferidos en ciclos posteriores (22), reportando una tasa de embarazo clínico y de recién nacido vivo de $35,9 \%$ y $21,8 \%$ respectivamente.

Actualmente, se considera que el número de niños sanos nacidos de MIV sobrepasa los 2000 (23) y hasta la fecha, no se han reportado informes alarmantes sobre la seguridad de la técnica (24). Además, Guzmán y cols demostraron que se pueden obtener embarazos sanos provenientes de folículos menores de $6 \mathrm{~mm}$ (25).

\section{CONCLUSIÓN}

La MIV ofrece algunas ventajas sobre los ciclos de reproducción asistida convencional, debido principalmente a la reducción de costos, uso limitado de gonadotrofinas, y la eliminación completa del riesgo de hiperestimulación ovárica (26). La MIV no debe ser comparada a las técnicas clásicas de FIV, sino que considerada como un tratamiento complementario a la FIV, especialmente para pacientes con alto riesgo de desarrollar SHO y pacientes oncológicas que deseen preservar su fertilidad.

\section{REFERENCIAS}

1. Edwards RG. Maturation in vitro of human ovarian oocytes. Lancet 1965;2(7419):926-9.

2. Yarali H, Zeyneloglu HB. Gonadotrophin treatment in patients with polycystic ovary syndrome. Reprod Biomed Online 2004;8(5):528-37.

3. Huang J, Chian R-C, Tan S. Ovarian hyperstimulation syndrome prevention strategies: in vitro maturation. Semin Reprod Med 2010;28(06):519-31.

4. Junk SM, Yeap D. Improved implantation and ongoing pregnancy rates after single-embryo transfer with an optimized protocol for in vitro oocyte maturation in women with polycystic ovaries and polycystic ovary syndrome. Fertil Steril 2012;98(4):888-92.

5. Lindenberg $\mathrm{S}$. New approach in patients with polycystic ovaries, lessons for everyone. Fertil Steril 2013;99(5):1170-2.

6. De Vos M, Ortega Hrepich C, Albuz FK, Guzman L, Polyzos NP, Smitz J, et al. Clinical outcome of nonhCG-primed oocyte in vitro maturation treatment in patients with polycystic ovaries and polycystic ovary syndrome. Fertil Steril 2011;96(4):860-4.

7. Le Du A, Kadoch IJ, Bourcigaux N, Doumerc S, Bourrier M-C, Chevalier N, et al. In vitro oocyte maturation for the treatment of infertility associated with polycystic ovarian syndrome: the French experience. Hum Reprod 2005;20(2):420-4.

8. Soderstrom-Anttila V. Favourable pregnancy results with insemination of in vitro matured oocytes from unstimulated patients. Hum Reprod 2005;20(6):153440.

9. Child TJ, Abdul-Jalil AK, Gulekli B, Tan SL. In vitro maturation and fertilization of oocytes from uns- 
timulated normal ovaries, polycystic ovaries, and women with polycystic ovary syndrome. Fertil Steril 2001;76(5):936-42.

10. Mikkelsen AL, Lindenberg S. Benefit of FSH priming of women with PCOS to the in vitro maturation procedure and the outcome: a randomized prospective study. Reproduction 2001;122(4):587-92.

11. Chian R-C, Buckett WM, Tulandi T, Tan SL. Prospective randomized study of human chorionic gonadotrophin priming before immature oocyte retrieval from unstimulated women with polycystic ovarian syndrome. Hum Reprod 2000;15(1):165-70.

12. Jurema MW, Nogueira D. In vitro maturation of human oocytes for assisted reproduction. Fertil Steril 2006;86(5):1277-91.

13. Fadini R, Canto MD, Renzini MM, Brambillasca F, Comi R, Fumagalli D, et al. Effect of different gonadotrophin priming on IVM of oocytes from women with normal ovaries: a prospective randomized study. Reprod Biomed Online 2009;19(3):343-51.

14. Fadini R, Mignini Renzini M, Dal Canto M, Epis A, Crippa M, Caliari I, et al. Oocyte in vitro maturation in normo-ovulatory women. Fertil Steril 2013;99(5):1162-9.

15. Chian R-C, Uzelac PS, Nargund G. In vitro maturation of human immature oocytes for fertility preservation. Fertil Steril 2013;99(5):1173-81.

16. Baerwald AR, Adams GP, Pierson RA. A new model for ovarian follicular development during the human menstrual cycle. Fertil Steril 2003;80(1):116-22.

17. Demirtas E, Elizur SE, Holzer H, Gidoni Y, Son W-Y, Chian R-C, et al. Immature oocyte retrieval in the luteal phase to preserve fertility in cancer patients. Reprod Biomed Online 2008;17(4):520-3.

18. Maman E, Meirow D, Brengauz M, Raanani H, Dor J, Hourvitz A. Luteal phase oocyte retrieval and in vitro maturation is an optional procedure for urgent fertility preservation. Fertil Steril 2011;95(1):64-7.
19. Albertini DF, Combelles CM, Benecchi E, Carabatsos MJ. Cellular basis for paracrine regulation of ovarian follicle development. Reproduction 2001;121(5):64753.

20. Son $\mathrm{W}-\mathrm{Y}$, Chung J-T, Demirtas E, Holzer H, Sylvestre $\mathrm{C}$, Buckett $\mathrm{W}$, et al. Comparison of in-vitro maturation cycles with and without in-vivo matured oocytes retrieved. Reprod Biomed Online 2008;17(1):59-67.

21. Guzman L, Ortega Hrepich C, Albuz FK, Verheyen G, Devroey P, Smitz J, et al. Developmental capacity of in vitro-matured human oocytes retrieved from polycystic ovary syndrome ovaries containing no follicles larger than $6 \mathrm{~mm}$. Fertil Steril 2012;98(2):503-7.e1-2.

22. Ortega Hrepich C, Stoop D, Guzman L, Van Landuyt $\mathrm{L}$, Tournaye $\mathrm{H}$, Smitz J, et al. A "freeze-all" embryo strategy after in vitro maturation: a novel approach in women with polycystic ovary syndrome? Fertil Steril 2013;100(4):1002-7.

23. Suikkari AM. In-vitro maturation: its role in fertility treatment. Curr Opin Obstet Gynecol 2008;20(3):2428.

24. Fadini R, Mignini Renzini M, Guarnieri T, Dal Canto M, De Ponti E, Sutcliffe A, et al. Comparison of the obstetric and perinatal outcomes of children conceived from in vitro or in vivo matured oocytes in in vitro maturation treatments with births from conventional ICSI cycles. Hum Reprod 2012;27(12):3601-8.

25. Guzman L, Adriaenssens T, Ortega-Hrepich C, Albuz FK, Mateizel I, Devroey P, et al. Human antral follicles $<6 \mathrm{~mm}$ : a comparison between in vivo maturation and in vitro maturation in non-hCG primed cycles using cumulus cell gene expression. Mol Hum Reprod 2012;19(1):7-16.

26. Shalom-Paz E, Almog B, Shehata F, Huang J, Holzer $\mathrm{H}$, Chian $\mathrm{R}-\mathrm{C}$, et al. Fertility preservation for breast-cancer patients using IVM followed by oocyte or embryo vitrification. Reprod Biomed Online 2010;21(4):566-71. 